easily done without recraniotomy. Autoradiography of the sources revealed satisfactory distribution of the exposure rate. These have maintained good quality after frequent use for animal experiments over ten months and leakage of the isotope was not observed.

The sources were inserted between duramater and cerebral cortex of normal adult cats in various period and histological and microangiographical examination were performed. On the strongest source containing of $8 \mathrm{mCi}$ of $\mathrm{Co} 60$, most of cats died in short period because of remarkable cerebral swelling associated with localized necrosis and bleeding under the source and diffuse demyelinization of subcortex of the irradiated portion. On the source containing of 3 or $1.3 \mathrm{mCi}$, however, only few cats died and the brain tissue reactions were mild even after enough irradiation in longer period.

\title{
d-3. Cytology of the Cerebrospinal Fluid
}

\author{
Mamoru Takagaki, Takizo Tomita, Minoru Ishit, Masatoshi Homma and \\ Tomohiro Matsuzawa \\ Department of Neurosurgery, Juntendo University School of Medicine
}

We have reported on the cytological study in the cerebrospinal fluid of the 159 patients with verified neoplasm of the central nervous system.

The specimens were collected during lumbar puncture, lumbar drainage, pneumoencepha'ography, ventricular drainage and puncture of the cystic tumors.

As the cell-catch procedure, centrifugation technique was used. The slides were stained with May-Giemsa, Haematoxylin-Eosin and fluorescent technique.

Of the 159 patients in the esries, the tumor cells were found in 34 cases of these CSF and in 9 cases of puncture of the cystic tumors.

The neoplasm must be in direct contact with the ventricles or subarachinoid space and it is necessary to exfoliate easily the tumor cells in the ventricles or subarachinoid space.

In the results of these series positive rate of tumor cells in the CSF is undoubtedly more frequent than in less malignancy of the tumor cells. In the mallignant tumor cells identification of the cellular characteristics more easy in less malignancy. In the identification of the atypical cells in the CSF, especially in the Papanicolau's class III, it is necessary to refer the clinical signs and symptoms of the petients. 